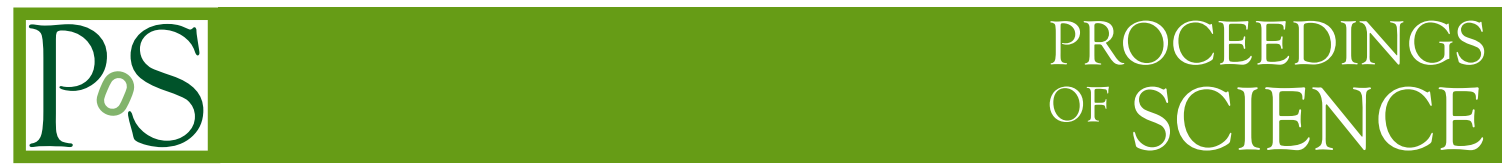

\title{
Attractor Structure of Gauged Nambu-Jona-Lasinio Model
}

\section{Hiroki Sakamoto*}

Department of Physics, Hiroshima University

E-mail: h-sakamoto@hiroshima-u.ac.jp

We have studied the inflation theory in the gauged Nambu-Jona-Lasinio model, which is motivated the alternative scenario to elementary scalar inflation theories like Higgs inflation. This model can produce the interesting values of the Cosmic Microwave Background fluctuations.

The 3rd International Symposium on âAIJQuest for the Origin of Particles and the Universe" 5-7 January 2017

Nagoya University, Japan

${ }^{*}$ Speaker. 


\section{Introduction}

The inflation theory has been introduced to solve some cosmological problems and its trace is presented in Cosmic Microwave Background (CMB) fluctuations. A lot of models are proposed to explain this fluctuations. Recently we can discriminate which models viable by precisely observation. Planck Satelite gives us the observational values of the CMB fluctuations [1]. In the slow-roll scenario, the inflation is controlled by the slow-roll parameter which is described to use the potential of the scalar field,

$$
\begin{aligned}
& \varepsilon \equiv \frac{1}{2 \kappa^{2}}\left(\frac{1}{V} \frac{\partial V}{\partial \phi}\right)^{2}, \\
& \eta \equiv \frac{1}{\kappa^{2}}\left(\frac{1}{V} \frac{\partial^{2} V}{\partial \phi^{2}}\right), \\
& \xi_{C M B} \equiv \frac{1}{\kappa^{4}} \frac{1}{V^{2}} \frac{\partial V}{\partial \phi} \frac{\partial^{3} V}{\partial \phi^{3}},
\end{aligned}
$$

where $\kappa^{2} \equiv 8 \pi G$. During the inflationary expansion, these parameters are less than unit. The expansion rate of the universe is given by the e-folding number,

$$
N=\kappa^{2} \int_{\phi_{\mathrm{end}}}^{\phi_{N}} d \phi \frac{V(\phi)}{\partial_{\phi} V}
$$

where $\phi_{N}$ means the value at the horizon crossing and $\phi_{\mathrm{end}}$ is the one at the end of the inflation. The e-folding number is required $N \sim 50-60$ to solve the horizon and flatness problem. CMB fluctuations are defined by the slow-roll parameters at the horizon crossing:

$$
\begin{aligned}
& n_{s}=1-6 \varepsilon+2 \eta, \\
& r=16 \varepsilon, \\
& \alpha_{s}=-24 \varepsilon^{2}+16 \varepsilon \eta-2 \xi_{C M B} .
\end{aligned}
$$

\section{Gauged Nambu-Jona-Lasinio inflation}

We have proposed the composite inflaton scenario where the composite inflaton is given by the fermion confinement [2]. For the one of such a model, we focus on the gauged Nambu-JonaLasinio (NJL) model. The Lagrangian is described by

$$
\mathscr{L}_{g N J L}=\mathscr{L}_{\text {gauge }}+\bar{\psi} i \hat{\not D} \psi+\frac{16 \pi^{2} g_{4}}{8 N_{f} N_{c} \Lambda^{2}}\left[(\bar{\psi} \psi)^{2}+\left(\bar{\psi} i \gamma_{5} \tau^{a} \psi\right)^{2}\right]
$$

where $\mathscr{L}_{\text {gauge }}$ shows $S U\left(N_{c}\right)$ pure gauge sector, $\hat{D}_{\mu}$ is the covariant derivative in curved space, $g_{4}$ is defined to the dimensionless four-fermion coupling, and, $\Lambda$ means the dynamical scale which is called compositeness scale. The fermion has $N_{f}$ flavors and the flavor symmetry is represented by the generators $\tau^{a}$. 
The corresponding theory to the gauged NJL model is known as the gauge-Higgs-Yukawa (gHY) theory,

$$
\begin{aligned}
\mathscr{L}_{g H Y}=-\frac{1}{4} F^{\mu v} F_{\mu \nu}+\frac{1}{2} \partial_{\mu} \sigma \partial^{\mu} \sigma+ & \frac{1}{2} \partial_{\mu} \pi^{a} \partial^{\mu} \pi^{a}-\frac{1}{2} m^{2}\left(\sigma^{2}+\pi^{a} \pi^{a}\right)-\frac{\lambda}{4}\left(\sigma^{2}+\pi^{a} \pi^{a}\right)^{2} \\
& -\frac{1}{2} \xi R\left(\sigma^{2}+\pi^{a} \pi^{a}\right)+\bar{\psi} i \hat{\not} \psi-y \bar{\psi}_{i}\left(\sigma+i \gamma_{5} \tau^{a} \pi^{a}\right) \psi .
\end{aligned}
$$

with the compositeness condition,

$$
\frac{1}{y^{2}\left(t_{\Lambda}\right)}=0, \quad \frac{\lambda\left(t_{\Lambda}\right)}{y^{4}\left(t_{\Lambda}\right)}=0, \quad \xi\left(t_{\Lambda}\right)=\frac{1}{6}, \quad \frac{m^{2}\left(t_{\Lambda}\right)}{y^{2}\left(t_{\Lambda}\right)}=\frac{2 a}{16 \pi^{2}} \Lambda^{2}\left(\frac{1}{g_{4}}-\frac{1}{\Omega\left(t_{\Lambda}\right)}\right),
$$

where $a \equiv 2 N_{f} N_{c}, \Omega\left(t_{\Lambda}\right)$ is a function contributed to the renormalization of the mass parameter by the gauge interaction, and $t_{\Lambda}=\left.\ln \left(\mu / \mu_{0}\right)\right|_{\mu=\Lambda}$ is a renormalization group (RG) parameter. Due to the compositeness condition (2.3), the gauged NJL model deformed by the auxilliary field method and gHY theory rescaled by $\sigma \rightarrow \sigma / y$ are equivalent at the $\mu=\Lambda$.

To be coincided both effective potential, we improve it RG invariantly. Here we assume the fixed gauge coupling, the large $\Lambda$ and no contribution from $\pi^{a}$. Then we can obtain the RG improved effective potential including the non-minimal scala-gravity interaction. The effective action of the gravity and scalar part is described by

$$
S_{J}=\int d^{4} x \sqrt{-g}\left[-\frac{\Omega^{2}}{2} R+\frac{1}{2} g^{\mu v} \partial_{\mu} \sigma \partial_{v} \sigma-U\right],
$$

where we use Planck Unit $\kappa^{2}=1$. Weyl factor and the effective potential is given by

$$
\begin{aligned}
& \Omega^{2}=1+\zeta_{1} \mu^{2-2 n} \sigma^{2 n}, \\
& U=\lambda_{1} \mu^{2} \sigma^{2}+\lambda_{2} \mu^{4-4 n} \sigma^{4 n},
\end{aligned}
$$

and $n=\left(1+3 \alpha_{g}\left(N_{c}^{2}-1\right) /\left(4 \pi N_{c}\right)\right)^{-1}$ is also free parameter $\left(\alpha_{g}=g^{2} / 4 \pi\right)$. The effective coupling $\lambda_{1}, \lambda_{2}$, and $\zeta_{1}$ are defined as follows:

$$
\begin{aligned}
& \lambda_{1}=\frac{1-n}{n G_{4 r}}, \\
& \lambda_{2}=\frac{3-2 n}{4 n}\left(\frac{a}{16 \pi^{2}} \frac{n}{1-n}\right)^{1-2 n}, \\
& \zeta_{1}=\frac{1}{6 n}\left(\frac{a}{16 \pi^{2}} \frac{n}{1-n}\right)^{1-n} .
\end{aligned}
$$

Due to the calculation of CMB fluctuations, we perform the conformal transformation $g_{\mu v} \rightarrow \tilde{g}_{\mu v}=$ $\Omega^{2}(x) g_{\mu \nu}$ and introduce a new scalar defined by

$$
\frac{d \phi}{d \sigma}=\sqrt{\frac{1}{\Omega^{2}}+\frac{3}{2}\left(\frac{1}{\Omega^{2}} \frac{\partial \Omega^{2}}{\partial \sigma}\right)^{2}}
$$

to get a canonical kinetic term, then we obtain

$$
S_{E}=\int d x^{4} \sqrt{-\tilde{g}}\left[-\frac{1}{2} \tilde{R}+\frac{1}{2} \tilde{g}^{\mu v} \partial_{\mu} \phi \partial_{\nu} \phi-V\right] .
$$




\section{Analysis of CMB fluctuations}

Evaluating the CMB fluctuations (1.7), we obtain three type solutions, so that the general chaotic inflation, $R^{2}$ inflation, and the another one. The chaotic inflation like solutions is given when we choice large $\alpha_{g} N_{c}$ with small $N_{f}$. Such a parameter region is beyond the scope of perturbative expansion, however, we expect that the fermion loop correction has no large contribution to CMB fluctuations in this region by the fixed gauge coupling. In this case we can choose $n$ arbitrarily, so that we obtain [3]

$$
n_{s}=\left\{\begin{array}{l}
1-\frac{2}{N} \\
1-\frac{2 n+1}{N}
\end{array} \quad r=\left\{\begin{array}{l}
\frac{8}{N} \\
\frac{16 n}{N}
\end{array} \quad \alpha_{s}= \begin{cases}-\frac{2}{N^{2}} & \left(2 n<1 \text { and } \lambda_{1} \neq 0\right), \\
-\frac{2 n+1}{N^{2}} & \left(2 n>1 \text { and } / \text { or } \lambda_{1}=0\right) .\end{cases}\right.\right.
$$

We notice that the large $\alpha_{g} N_{c}$ limit means the limit of $n \rightarrow 0$ but the small $n$ enhance the mass term. Then the small $n$ limit predict the specific prediction, namely $\phi^{2}$ chaotic inflation, not but the general one.

If we consider the large $N_{f}$ limit, the prediction of CMB fluctuations correspond to the ones of Higgs inflaion or $R^{2}$ inflation. It is caused by the large effective non-minimal coupling (2.9). The Weyl factor can be written by the canonical scalar $\phi$, and if the mass term can drop, the potential in Einstein frame $V_{E}$ is described by

$$
V_{E}=\frac{U}{\Omega^{4}}=\hat{\lambda}(1-\exp (-\sqrt{2 / 3} \phi))^{2}
$$

where $\hat{\lambda}$ is an effective coupling defined by

$$
\hat{\lambda} \equiv \frac{\lambda_{2}}{\zeta_{1}^{2}}=\frac{144 \pi^{2}}{a}(3-2 n)(1-n),
$$

As is known, such a potential gives the famous prediction of CMB fluctuations,

$$
n_{s} \sim 1-\frac{2}{N}, \quad r \sim \frac{12}{N^{2}}, \quad \alpha_{s} \sim-\frac{2}{N^{2}},
$$

and they predict the suitable fluctuations.

The weak gauge coupling limit, $g \rightarrow 0$, give us interesting results. The effective potential $U$ and Weyl factor $\Omega^{2}$ in this limit is rewritten as follows:

$$
\begin{aligned}
& U=\frac{3 \alpha_{g}\left(N_{c}^{2}-1\right)}{4 \pi N_{c}}\left[\frac{\mu^{2}}{G_{4 r}} \sigma^{2}+\frac{4 \pi^{2}}{a} \sigma^{4}\right]+O\left(\alpha_{g}^{2}\right) \\
& \Omega^{2}=1+\frac{1}{6}\left[1+\frac{3 \alpha_{g}\left(N_{c}^{2}-1\right)}{4 \pi N_{c}}\left(1-\ln \left(\frac{a N_{c} \mu^{2}}{12 \pi \alpha_{g}\left(N_{c}^{2}-1\right) \sigma^{2}}\right)\right)\right] \sigma^{2}+O\left(\alpha_{g}^{2}\right) .
\end{aligned}
$$

From these expression, we can analytically evaluate CMB fluctuation,

$$
n_{s} \sim 1-\frac{2}{N}, \quad r \sim \frac{24}{N^{2}}, \quad \alpha_{s} \sim-\frac{2}{N^{2}},
$$

especially the tensor-to-scalar ratio $r$ has comparable value with Higgs inflation. 


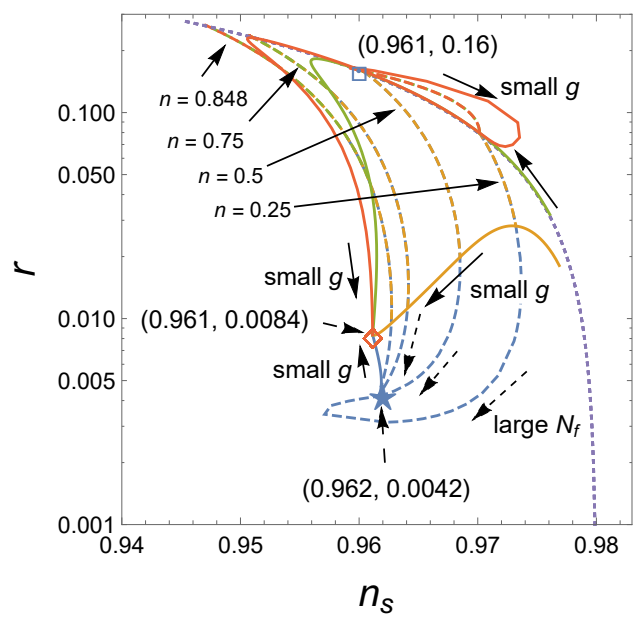

Figure 1: The attractor behavior of $g$ (solid line) and $N_{f}$ (dashed line) in $n_{s}-r$ plane for $G_{4 r}=10^{10}$. Two fixed point $\star$ and $\diamond$ are consistent for the observation from Planck.

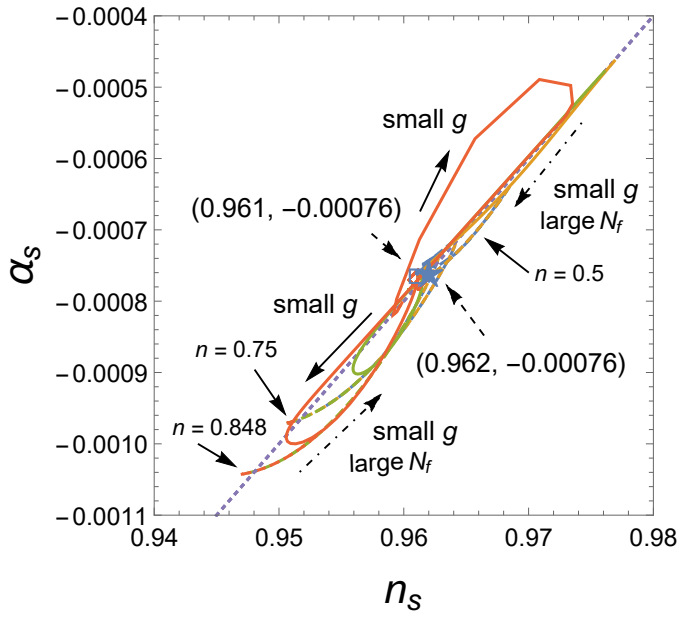

Figure 2: The attractor behavior of $g$ (solid line) and $N_{f}$ (dashed line) in $n_{s}-\alpha_{s}$ plane for $G_{4 r}=$ $10^{10}$. The fixed points predicts almost same value.

\begin{tabular}{l|lll} 
& $n_{s}$ & $r$ & $\alpha_{s}$ \\
\hline \hline$n \rightarrow 0$ & 0.961 & 0.16 & -0.0076 \\
large $N_{f}$ & 0.962 & 0.0042 & -0.0076 \\
small $g$ & 0.961 & 0.0084 & -0.0076 \\
\hline observation & $0.9645 \pm 0.0049$ & $<0.1$ & $-0.0057 \pm 0.0071$
\end{tabular}

Table 1: The prediction of each limit and the observational value of CMB fluctuations [1].

From above analysis, it is found that CMB fluctuations evaluated from gauged NJL inflation have two attractor behavior. Such behaviors are shown in Fig. 1 and Fig. 2. The symbols $\square$, $\star$, and $\diamond$ means the prediction of $\phi^{2}$ inflation, $R^{2}$ inflation, and the gauged NJL inflation in weak gauge coupling limit. The dotted line shows the general prediction of chaotic inflation. The behavior of varying $N_{f}$ is depicted by the dashed line. The prediction of $R^{2}$ inflation can be reproduced in sufficient large $N_{f}$. This attractor behavior corresponds to the one of Higgs inflation. The solid lines denote the behavior for $g$ dependence. It is found such a attractor behavior can be distinguished the one of the Higgs inflation. The numerical results of these attractor points are in Tab. 1 and we find the different CMB fluctuations of each limit.

\section{Conclusion}

We have studied the CMB fluctuations of the gauged NJL inflation as a candidate of a realistic inflation theory. In this model some interesting limits exist. The predictions of CMB fluctuations are explicitly expressed by (3.4) and (3.7). The attractor behavior is found and shown in Fig. 1 and Fig. 2. Especially, the attractor with respect to the gauge coupling and its small limit characterizes the NJL inflation. 


\section{Acknowledgments}

The main part of this talk is based on the work collaborated with Tomohiro Inagaki and Sergei D. Odintsov.

\section{References}

[1] P. A. R. Ade et al. [Planck Collaboration], Planck 2015 results. XIII. Cosmological parameters, Astron. Astrophys. 594, A13 (2016) doi:10.1051/0004-6361/201525830 [arXiv: 1502.01589 [astro-ph.CO]].

[2] T. Inagaki, S. D. Odintsov and H. Sakamoto, Gauged Nambu-Jona-Lasinio inflation, Astrophys. Space Sci. 360, no. 2, 67 (2015) doi:10.1007/s10509-015-2584-0 [arXiv:1509.03738 [hep-th] ].

[3] T. Inagaki, S. D. Odintsov and H. Sakamoto, Inflation from the finite scale gauged NambuâĂŞJona-Lasinio model, Nucl. Phys. B 919, 297 (2017) doi:10.1016/j.nuclphysb.2017.03.024 [arXiv:1611.00210 [hep-ph]]. 